\title{
Characterization of Nonlinear Finger Pad Mechanics for Tactile Rendering
}

\author{
Eder Miguel $^{1}$, Maria Laura D'Angelo ${ }^{2}$, Ferdinando Cannella ${ }^{2}$, Matteo Bianchi ${ }^{2,3}$, \\ Mariacarla Memeo ${ }^{2}$, Antonio Bicchi ${ }^{3}$, Darwin G. Caldwell ${ }^{2}$ and Miguel A. Otaduy ${ }^{1}$
}

\begin{abstract}
The computation of skin forces and deformations for tactile rendering requires an accurate model of the extremely nonlinear behavior of the skin. In this work, we investigate the characterization of finger mechanics with the goal of designing accurate nonlinear models for tactile rendering. First, we describe a measurement setup that enables the acquisition of contact force and contact area in the context of controlled finger indentation experiments. Second, we describe an optimization procedure that estimates the parameters of strain-limiting deformation models that match best the acquired data. We show that the acquisition setup allows the measurement of force and area information with high repeatability, and the estimation method reaches nonlinear models that match the measured data with high accuracy.
\end{abstract}

\section{INTRODUCTION}

The field of haptics is experiencing a growth of tactile display technology, in particular wearable devices that render haptic interaction directly on the user's skin [18], [12], [31], [29], [25]. In this context, tactile rendering gains relevance as the technology for computing the contact forces and/or deformations that mimic those occurring with a virtual object, and which are then displayed to the user. The correct estimation of such forces and/or deformations requires an accurate model of the interaction between the skin and the virtual objects.

Skin is extremely nonlinear [28], [4]. It is very soft under small forces, and in this way mechanoreceptors are excited even under small actions. But skin soon becomes almost rigid under moderate compression. Better understanding of the sense of touch, intelligent design of tactile devices, and accurate tactile rendering, all call for skin simulation methods that capture its extreme nonlinear behavior robustly and efficiently.

Strain-limiting models [26], [33] appear as an attractive means for simulating such nonlinearities both robustly and efficiently. Perez et al. [23] demonstrated that strain-limiting models can be used for finger simulation in the context of haptic rendering, and succeeded to match the extremely nonlinear force-area relationship observed experimentally [28]. But their simulation models were designed ad-hoc, with no connection to the behavior of a particular user's skin.

In this work, we address a preliminary characterization of nonlinear finger pad mechanics and the estimation of nonlinear deformation models suited for tactile rendering. We propose both an acquisition setup and an estimation method

\footnotetext{
${ }^{1}$ URJC Madrid, c/ Tulipán s/n, 28933 Móstoles (Madrid), Spain

2 Istituto Italiano di Tecnologia, Via Morego 30, 16163 Genova, Italy

${ }^{3}$ University of Pisa, Italy
}

that are integrated for the design of accurate, user-specific simulation models.

The acquisition setup enables the combined acquisition of contact force and contact area, which characterize the nonlinear behavior of the skin. We have integrated force and vision sensors with actuators that allow the indentation of the finger pad under controlled displacements and velocities. The estimation method optimizes the parameters of an energybased strain-limiting model [24]. The difference between simulated and estimated measurements is minimized in a simulated replica of the acquisition experiment.

We show that the acquisition setup allows the measurement of force and area information with high repeatability, and the estimation method reaches nonlinear models that match the measured data with high accuracy.

\section{RELATED WORK}

Two of the most common deformation models used in computer graphics and haptic rendering are the linear corotational model [19] and the St. Venant-Kirchhoff model [14]. However, finger tissue modeling requires nonlinear deformation models, such as Ogden, Neo-Hookean or MooneyRivlin hyperelasticity models [20]. Many numerical models of the finger pad have been built, which differ in terms of dimensionality, accuracy and mechanical response to different tasks (see e.g. [36], [16], [8], [10] among the most significant).

The main drawback of these hyperelasticity models is that they are extremely nonlinear and therefore produce very high numerical stiffness. As a consequence, stable simulation requires tiny timesteps, which reduces the range of applications that can benefit from these deformation models. On the other hand, strain or energy-limiting approaches [26], [33] are able to capture such extreme nonlinearities while still allowing large timesteps.

In addition to a deformation model capable of capturing the nonlinear behavior of finger pad mechanics, appropriate model parameters must be found that reproduce the behavior of each person's fingers. Pai et al. [21] presented both an estimation methodology and a measurement system for linear elasticity estimation. More recently, parameter estimation has been applied to a wide variety of deformation effects, such as heterogeneity [3], nonlinearities in cloth [35] or even viscoelasticity [27]. Other works have explored solutions to the estimation problem using different types of input data, such as captured video sequences with little control over boundary conditions [2], [5], or more controlled setups 


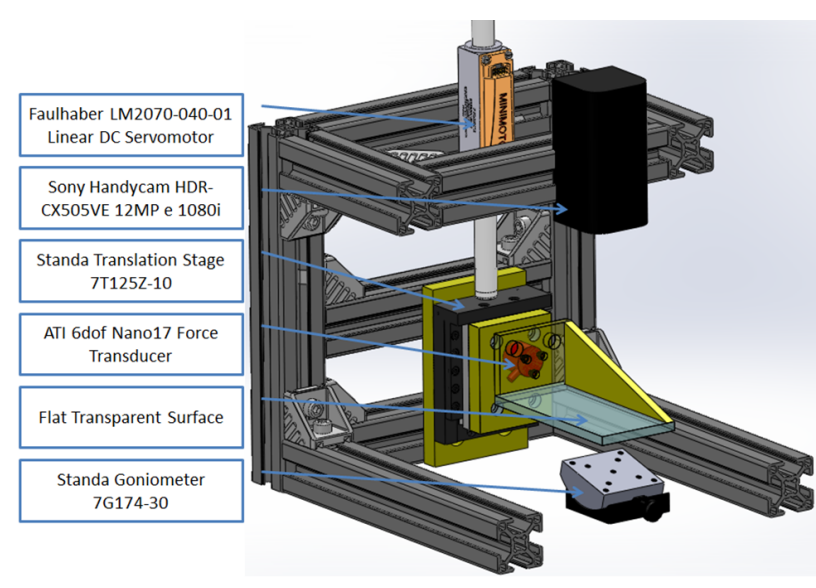

Fig. 1. Components of the measurement apparatus.

with computer-vision-based tracking, such as [17]. Ahn et al. [1] present a measurement and estimation system that is able to produce parameter estimations for soft-tissues using Neo-Hookean models, but limit those estimations to small deformations, where nonlinearities are subtle and do not pose major challenges in numerical simulation. In a more recent work by Goksel et al. [13], they propose a system to estimate both elasticity parameters and surface geometry from position-force readings, but they apply it to linear isotropic elasticity models.

In-vivo measurement of mechanical properties of skin may also follow different approaches (see e.g. [9], [32], [36], [16]), based on diverse acquisition systems, such as digital camera-based systems [15], Magnetic Resonance (MR) images taken before and during compressional loading of the finger tissue [6], or non-intrusive suction instruments [11], among others. The type of measured data suitable for parameter estimation of finger mechanics may be based on one of three basic strategies: (i) force-displacement data and deformation under line load [8], [10]; (ii) force-area measurements [30]; or (iii) pressure measurements at the contact [22].

\section{Measurement System And Data}

As discussed in the introduction, the estimation of a skin model for tactile rendering requires force-deformation data that excites the nonlinear mechanics of the finger pad. In this section, we describe a measurement apparatus for the acquisition of contact force and contact area during normal indentation of the finger pad. These two variables are known to exhibit an extremely nonlinear relationship due to the saturation of finger pad deformation [28]. We also describe the acquisition procedure, and we list the data obtained for a test subject.

\section{A. Measurement Apparatus}

The test rig is a one degree of freedom (DoF) device that moves a flat surface (i.e., the indenter) towards the finger pad, which is fixed, in order to indent it. The components of the experimental system are shown in Fig. 1. The displacement
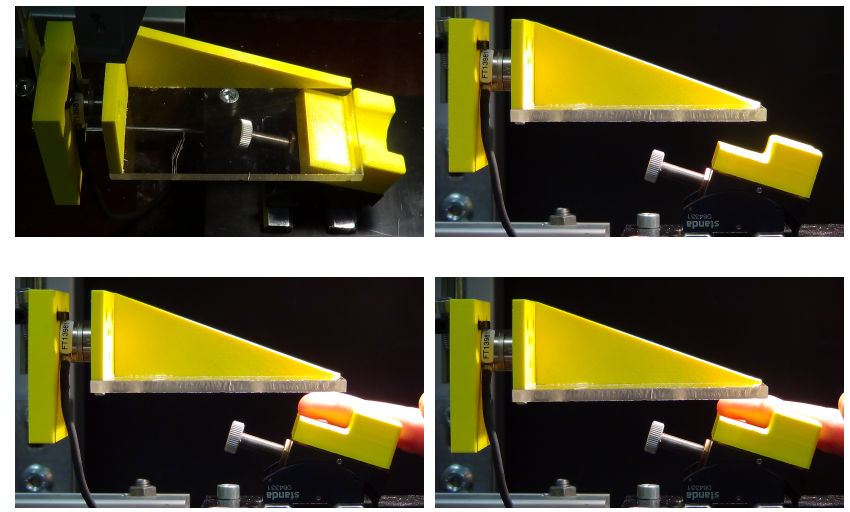

Fig. 2. Top: Two views of the indenter and the finger holder. Bottom Demonstration of finger deformation during an acquisition experiment, as the indenter surface is moved down toward the finger.

is given by a linear DC actuator (Faulhaber LM2070-040-01 plus MCLM3003 controller) with $9.2 \mathrm{~N}$ of constant force, $94 \mathrm{~m} / \mathrm{s}^{2}, 200 \mu \mathrm{m}$ of accuracy and $60 \mu \mathrm{m}$ of repeatability. The one directional motion is guaranteed by the slider (Standa 7T125Z-10), which has an angular deviation of less than $200 \mu \mathrm{rad}$, and the structure was stiffened with a side rib, as shown in Fig. 1. To correctly position the finger and control its orientation during the experimental test, we used a micro goniometer (Standa 7G174-30), with reading resolution of $0.1 \mathrm{deg}$

Forces and torques are recorded using a 6-DoF force/torque sensor (ATI 6-DoF Nano17) with $0.00625 \mathrm{~N}$ of force resolution and $0.03125 \mathrm{Nmm}$ of torque resolution. The force/torque sensor, rigidly connected to the indenter surface, is able to measure the forces along 6 directions in order to prevent any misalignment of the fingertip and the plate. As a result, it produces a very accurate assessment of the vertical force.

The contact area is acquired visually using a camera. For this purpose, the indenter surface was built transparent in Plexiglass. We have used a Sony HDR-CX505VE camera, with $1500 \times 1120$ effective pixels per video frame and $50 \mathrm{fps}$ acquisition rate. This high resolution enables more accurate area estimates than previous test rigs [28]. To measure the contact area, we apply a post-processing step in Matlab. First, we segment the fingertip contact area based on a luminance threshold, which is selected manually for each subject's data set. The skin in contact appears brighter, hence all the pixels above the luminance threshold are segmented. Finally, the contact area value is estimated simply by counting the number of segmented pixels and multiplying the result by the surface area corresponding to each pixel.

The compliance of the structure was validated through numerical simulations. The compliance between the camera and the flat surface was smaller than $0.1 \mathrm{~mm}$, which leads to a misalignment between the video camera (rigidly connected to the structure) and the finger pad of less than $0.25 \mathrm{deg}$. The maximum displacement in the structure is of $0.30 \mathrm{~mm}$, which is within the focus field of the camera, and leads to a variation in pixel size of just $0.027 \%$. Therefore, we can 

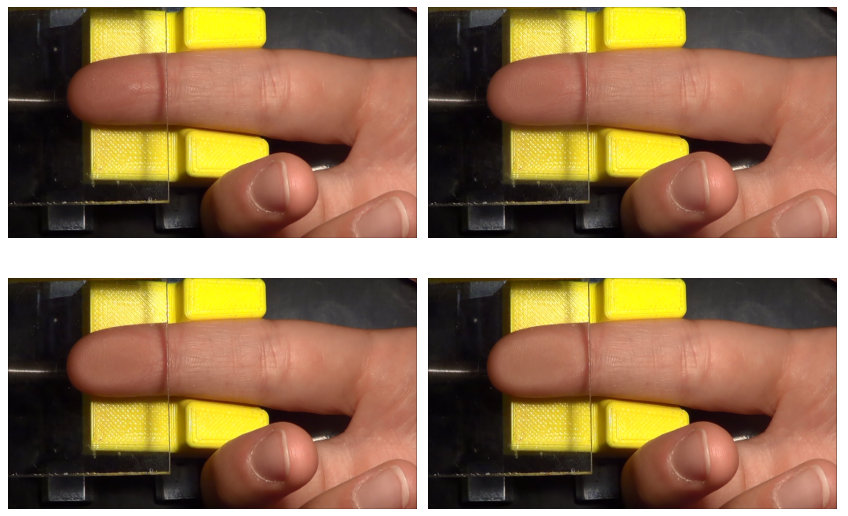

Fig. 3. Contact area under different indentations. From left to right and top to bottom: $1 \mathrm{~mm}, 2 \mathrm{~mm}, 3 \mathrm{~mm}$ and $4 \mathrm{~mm}$.

safely conclude that the influence of the compliance of the structure on the acquired image is negligible.

\section{B. Acquisition Procedure}

For the acquisition experiments, the subject placed the finger pad on the finger-holder, and the indenter surface was moved toward the finger pad, as shown in Fig. 2. To prevent losing contact during the experiment, the finger was fixed to the finger-holder on the top of the nail. The finger pad was oriented at a $15 \mathrm{deg}$ angle w.r.t. the indenter surface.

The experiments consisted in moving the indenter according to a step velocity function up to a certain displacement, then wait for one second to allow relaxation of the finger, and finally move the indenter back again. During this process, using the force sensor and the video-camera we record the forces, torques and contact area, respectively. The linear actuator can control both the speed and displacement of the indenter. With the experimental data, we wish to estimate only the elastic properties of the finger pad skin, disregarding effects such as viscosity. For this reason, to minimize the effect of viscosity we have selected a very low velocity for the indenter $(2 \mathrm{~mm} / \mathrm{s})$.

\section{Experimental Data}

Force-area data was acquired for the index finger pad of a right-handed student female (28 years old). The participant gave her informed consent to the experiments, and her finger pad was free of calluses.

We acquired data for 4 different maximum displacements of the indenter $(1,2,3$, and $4 \mathrm{~mm})$, with 3 repetitions, all of them with an indenter velocity of $2 \mathrm{~mm} / \mathrm{s}$. Fig. 3 shows some of the images captured with the camera, with different contact area per indenter displacement. Similarly, Fig. 4 shows force measurements over time for several indentation cycles with a maximum indentation of $4 \mathrm{~mm}$ and an indentation speed of $2 \mathrm{~mm} / \mathrm{s}$.

We have measured the peak area and force values for all the indentations in all 3 repetitions. The measured area values, along with the mean area across repetitions and the standard deviation, are reported in Table I. The measured

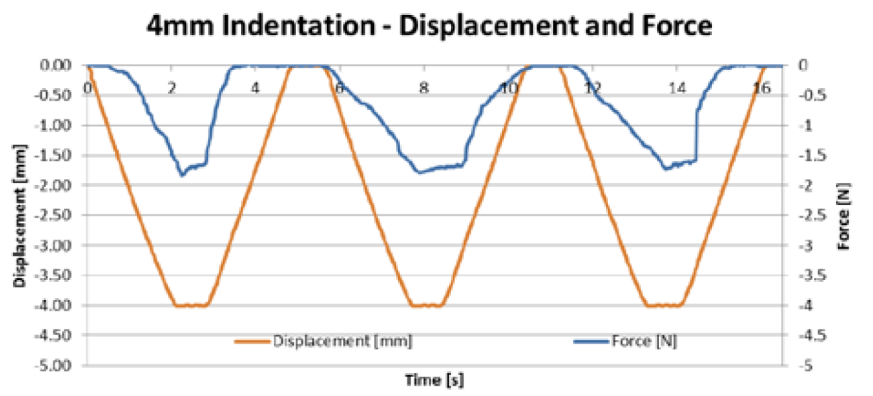

Fig. 4. Force and displacement measurement over time for maximum indentations of $4 \mathrm{~mm}$.

\begin{tabular}{|c|c|c|c|c|}
\hline \multicolumn{5}{|c|}{ Experimental Area Values $\left[\mathrm{mm}^{2}\right]$} \\
\hline Indentation & $1 \mathrm{~mm}$ & $2 \mathrm{~mm}$ & $3 \mathrm{~mm}$ & $4 \mathrm{~mm}$ \\
\hline Test 1 & 92.82 & $(148.8)$ & 147.2 & 223.7 \\
\hline Test 2 & 75.36 & 117.8 & 129.6 & 205.9 \\
\hline Test 3 & 99.19 & 106.6 & 121.6 & 182.2 \\
\hline Average & 89.12 & 124.6 & 132.8 & 203.93 \\
\hline STD & 12.34 & 21.86 & 13.10 & 20.82 \\
\hline
\end{tabular}

TABLE I

CONTACT AREAS MEASURED AT 4 DIFFERENT INDENTATIONS WITH 3 REPETITIONS EACH.

\begin{tabular}{|c|c|c|c|c|}
\hline \multicolumn{5}{|c|}{ Experimental Force Values [N] } \\
\hline Indentation & $1 \mathrm{~mm}$ & $2 \mathrm{~mm}$ & $3 \mathrm{~mm}$ & $4 \mathrm{~mm}$ \\
\hline Test 1 & 0.80 & $(1.01)$ & 1.72 & 3.36 \\
\hline Test 2 & 0.75 & 1.05 & 1.76 & 3.59 \\
\hline Test 3 & 0.78 & 1.02 & 1.64 & 3.45 \\
\hline Average & 0.78 & 1.03 & 1.71 & 3.47 \\
\hline STD & 0.03 & 0.02 & 0.06 & 0.12 \\
\hline
\end{tabular}

TABLE II

CONTACT FORCES MEASURED AT 4 DIFFERENT INDENTATIONS WITH 3 REPETITIONS EACH.

force values, along with the mean force across repetitions and the standard deviation, are reported in Table II.

After analyzing the acquired data, it appears of high repeatability. We have identified two small issues that could be improved in future versions of the setup. First, one of the force values, the first repetition at $2 \mathrm{~mm}$ displacement, appears as an outlier, and was discarded for model estimation. Second, despite the low indentation velocity, the peak forces may exhibit a small viscous effect, as the contact force decreases a bit when the indenter stops moving.

\section{Nonlinear Model Estimation}

In this section we present the deformation model and the parameter estimation procedure that we used to reproduce the behavior of finger skin.

\section{A. Deformation Model}

As our base deformation model, we use a continuum elasticity model discretized with the Finite Element Method (FEM). Specifically, we adopt a standard St. VenantKirchhoff (StVK) model with a tetrahedral discretization. The StVK model is defined in terms of the energy-density 

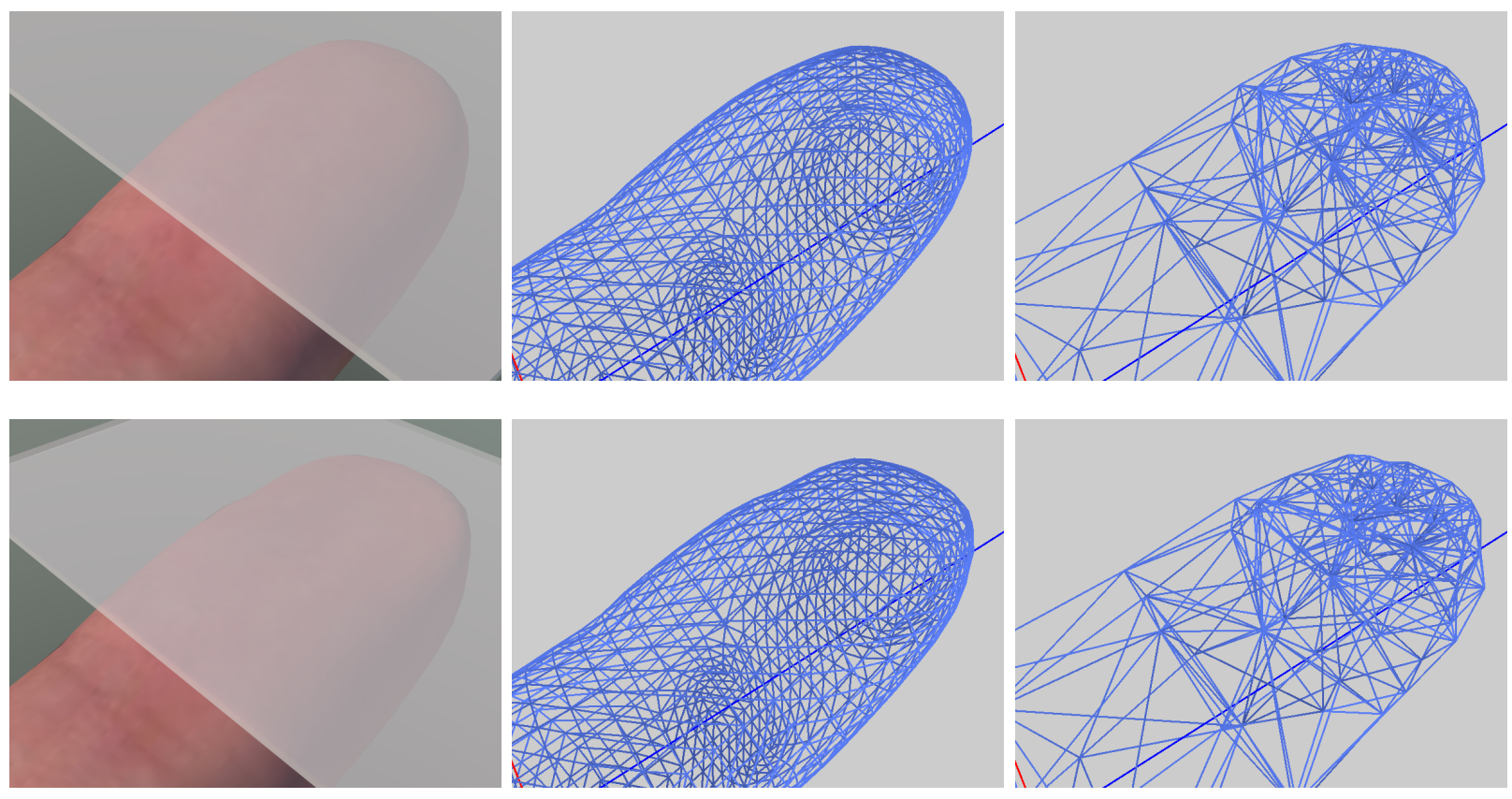

Fig. 5. The images show two simulation steps of the finger in contact with the indenter surface during the estimation procedure. On top, in the undeformed configuration, and on the bottom, deformed with a contact area of $150 \mathrm{~mm}^{2}$. The differences are subtle, but so is the deformation of real finger pads, due to their extreme nonlinearity. Left: full render of the finger and the indenter; Middle: triangle mesh of the finger used for contact handling; Right: tetrahedral mesh of the finger used for deformation.

function:

$$
\Psi=\mu \operatorname{tr}\left(\mathbf{E}^{2}\right)+\frac{1}{2} \lambda \operatorname{tr}(\mathbf{E})^{2}
$$

where $\mathbf{E}$ is the Green-strain tensor:

$$
\mathbf{E}=\frac{1}{2}\left(\mathbf{F}^{T} \mathbf{F}-\mathbf{I}\right)
$$

and $\mathbf{F}$ is the deformation gradient.

We extend this model with extreme nonlinearity, modeled using energy-limiting constraints [24]. Energy constraints are defined for each discretization element as:

$$
C_{e}=1-\frac{\Psi_{e}}{\Psi_{\max }} \geq 0
$$

where $\Psi_{e}$ is the energy density computed for the discretization element and $\Psi_{\max }$ is the maximum allowed energy density. This method for capturing nonlinearities is very flexible and could be applied to any constitutive model and discretization.

For a tetrahedral discretization, the energy density is constant inside each tetrahedron. For other types of discretizations, such as hexahedral discretization, we propose to estimate each element's energy using quadrature integration, and then divide this amount by the element's volume to compute an average energy density per element.

We use the deformation model in the context of both dynamic simulation and static deformation. And in both cases, regular simulation methods need to be extended to accommodate energy-limiting constraints. For dynamic simulation, we enforce the energy-limiting constraints on every simulation step through Lagrange multipliers and a Linear Complementarity Problem (LCP) solver [7]. For static equilibrium, we formulate the problem as the minimization of the potential energy subject to the energy-limiting constraints, and solve it using dynamic relaxation [34].

\section{B. Estimation Procedure}

Our goal is to reproduce the behavior observed in the measurements using our simulation framework. In order to achieve this goal, we replicate the experimental setup and simulate the response of a virtual finger being compressed by a flat surface. We aim for the simulated contact forces to be as similar as possible to the experimental ones. However, before we can produce a realistic behavior of the simulated finger, we need to determine the parameters of the deformation model that produce an appropriate response.

We formulate this estimation process as an optimization problem where we try to minimize the difference between experimental and simulated contact forces at experimental contact areas. The corresponding objective function can be expressed as:

$$
f_{o b j}=\sum_{i=1}^{N}\left\|\mathbf{f}_{i}-\overline{\mathbf{f}}_{i}\right\|^{2}
$$

where $\overline{\mathbf{f}}_{i}$ is the $i$-th experimental measured force, $\mathbf{f}_{i}$ is the corresponding simulated force acting on the flat surface pressing the fingertip and $N$ is the number of input measurements.

In order to obtain the simulated contact force values we built a simulation framework that allows us to simulate the behavior of the finger using FEM. The flat surface used in the experimental setup is represented by a plane of the same dimensions. For the finger, we use a high resolution surface mesh (2656 triangles) to handle collision detection and response and to compute the contact area with the plane, and a coarser tetrahedral mesh (347 tetrahedra) for FEM 
simulations. To evaluate the contact area at any time in the simulation, we sum the exact area of the triangle surface mesh that is closer than $10 \mu \mathrm{m}$ from the indenter plane.

We replicate the boundary conditions of the experimental setup by positioning the finger in the same configuration and fixing the bottom nodes. During simulation, contact forces between the plane and the finger are computed using penalty forces with a stiffness value large enough to guarantee penetrations smaller than $10 \mu \mathrm{m}$. Fig. 5 shows the finger model in its undeformed state and once deformed.

Due to the discretization, we cannot match the experimental contact areas exactly, so we apply an alternative approach to compute the simulated contact forces $\mathbf{f}_{i}$. First, we compute the simulated contact force and contact area at progressively increasing indentation values in the range of the experimental ones, always ensuring that all segments between input indentations are sampled. Then, we interpolate the simulated contact forces at the experimental contact areas obtaining $\mathbf{f}_{i}$, which allows us to evaluate the objetive function.

Since the viscosity of the finger is unknown, we completely eliminate its effect in the simulated setup by solving finger deformations under static equilibrium. We found that the solution of such static equilibria performed best by using dynamic relaxation. Therefore, we perform dynamic solves using Backward Euler time integration with a single Newton step. Then, we monitor the kinetic energy and set velocities to zero whenever a maximum is reached. In this context of dynamic solves, energy-limiting constraints are enforced using Lagrange multipliers and an LCP solver.

Finally, we define the variables to estimate. We apply a two-step estimation process in which we first estimate Young's modulus for the linear StVK constitutive model with Poisson ratio $v=0.45$. Then, we add the energylimiting constraint and re-estimate Young's modulus and the maximum energy value of the energy-limiting constraint, $\Psi_{\max }$ in Eq. (3).

\section{Results}

In order to apply our estimation pipeline, we first defined the set of input experimental measurements. Specifically, we removed the outliers from the force-area pairs in Table I and Table II (data values corresponding to Test 1 and $2 \mathrm{~mm}$ indentation, in parenthesis) and ran our estimation pipeline as described above. For a linear StVK, we obtained an optimal Young's modulus $E=53.7 \mathrm{kPa}$. For the energy-limiting StVK model, the optimal Young's modulus was reduced to $E=41.9 \mathrm{kPa}$, and the energy density constraint was estimated as $\Psi_{\max }=2708$. For a one-dimensional deformation the energy density can be computed as $\Psi=\frac{1}{2} E\|\varepsilon\|^{2}$. Under this assumption, the energy density constraint corresponds to a maximum allowed strain of $36 \%$.

In Fig. 6 we show the contact area vs. contact force evaluation plot. With the purely linear model, the average force error was of $27.5 \%$, but with energy-limiting constraints this error was reduced to $16.2 \%$. The purely linear model averages the behavior for small and large contact

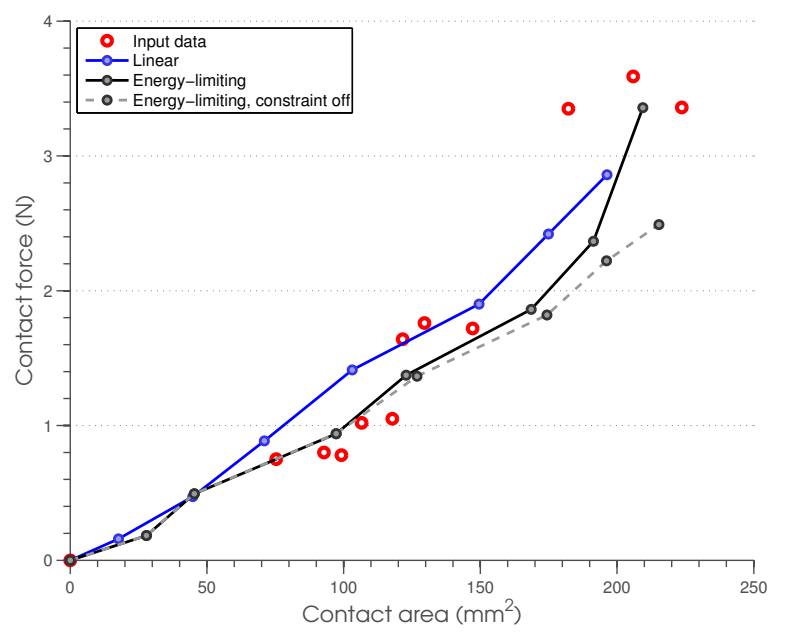

Fig. 6. Evaluation of contact area vs. contact force for the estimated linear and energy-limiting models, as well as for the linear component of the second one.

areas, but it appears too stiff for small contact areas and too soft as the contact area grows. The nonlinear model using energy-limiting constraints, instead, effectively captures the highly nonlinear behavior. We can also see how the nonlinear model separates from its linear component when constraints are activated around $100 \mathrm{~mm}^{2}$ contact area, and how the difference becomes larger as the contact area approaches $200 \mathrm{~mm}^{2}$.

The performance of the estimation pipeline depends on the number of degrees of freedom in the estimation process, growing with the number of parameters to estimate. In our case, for the linear estimation step it took approximately 3 hours to obtain the estimated Young's modulus, while for the nonlinear step it took about 14 hours for the optimization algorithm to find Young's modulus and the energy-limiting constraint value $\Psi_{\max }$. The bottleneck in this process was the solution of static equilibria under energy-limiting constraints and very high contact stiffness, which required small time steps. In our experiment we did not suffer convergence problems. With the amount of data available and the small number of parameters to estimate, the objective function was sufficiently regularized and appeared smooth. In addition, the progressive estimation, solving first for Young's modulus alone, and then using this result to initialize the search for both Young's modulus and the energy limit together, accelerates convergence and helps avoid local minima.

\section{DISCUSSION}

This paper presents a preliminary integrated approach to measure and model skin properties. Results are encouraging, and demonstrate that an efficient and robust nonlinear model of skin mechanics can be estimated from controlled force and area measurements.

Both the model and the evaluation of the method present limitations, but these can be addressed in the future by extending the current approach. Concerning the evaluation, it will be interesting to increase the number of measurements, 
but in particularly the number of test subjects. This will allow investigating inter-subject variability of the model parameters. One exciting avenue of future work would be to develop a generic model of nonlinear skin mechanics, perhaps modulated by a few parameters such as age and gender.

Concerning the model, higher accuracy would require the extension to other material properties, such as viscosity and friction, as well as material heterogeneity. But extending the model in this way will also require the design of novel measurement setups and estimation procedures.

\section{ACKNOWLEDGEMENTS}

This work was supported in part by grants from the Spanish Ministry of Economy (TIN2012-35840), the European Research Council (ERC Advanced Grant no. 291166 SoftHands and ERC Starting Grant no. 280135 Animetrics), and the EU FP7 (project no. 601165 WEARHAP and project no. 248587 The Hand Embodied).

\section{REFERENCES}

[1] B. Ahn and J. Kim. An efficient soft tissue characterization method for haptic rendering of soft tissue deformation in medical simulation. In Frontiers in the Convergence of Bioscience and Information Technologies, 2007. FBIT 2007, pages 549-553. IEEE, 2007.

[2] K. S. Bhat, C. D. Twigg, J. K. Hodgins, P. K. Khosla, Z. Popović, and S. M. Seitz. Estimating cloth simulation parameters from video. In Proc. ACM SIGGRAPH/Eurographics SCA, pages 37-51, 2003.

[3] B. Bickel, M. Bächer, M. A. Otaduy, W. Matusik, H. Pfister, and M. Gross. Capture and modeling of non-linear heterogeneous soft tissue. ACM Trans. Graph., 28(3):89:1-89:9, July 2009.

[4] J. E. Bischoff, E. M. Arruda, and K. Grosh. Finite element modeling of human skin using an isotropic, nonlinear elastic constitutive model. J Biomech, 33(6):645-652, Jun 2000.

[5] K. Bouman, B. Xiao, P. Battaglia, and W. Freeman. Estimating the material properties of fabric from video. In Computer Vision (ICCV), 2013 IEEE International Conference on, pages 1984-1991, 2013.

[6] A. Bowden, R. Rabbitt, and J. Weiss. Stress and strain in the human distal phalanx under indentation. In [Engineering in Medicine and Biology, volume 1, 1999.

[7] R. Cottle, J. Pang, and R. Stone. The Linear Complementarity Problem. Academic Press, 1992.

[8] K. Dandekar, B. I. Raju, and M. A. Srinivasan. 3-D finite-element models of human and monkey fingertips to investigate the mechanics of tactile sense. J Biomech Eng, 125(5):682-691, Oct 2003.

[9] H. Fruhstorfer, U. Abel, C. D. Garthe, and A. Knuttel. Thickness of the stratum corneum of the volar fingertips. Clin. Anat., 13(6):429-433, 2000.

[10] G. J. Gerling, I. I. Rivest, D. R. Lesniak, J. R. Scanlon, and L. Wan. Validating a population model of tactile mechanotransduction of slowly adapting type I afferents at levels of skin mechanics, singleunit response and psychophysics. IEEE Trans Haptics, 7(2):216-228, 2014.

[11] S. Giavazzi, M. F. Ganatea, M. Trkov, P. Šuštarič, and T. Rodič. Inverse determination of viscoelastic properties of human fingertip skin. $R M Z$ ? Materials and Geoenvironment, 57(1):1?16, 2010.

[12] B. Gleeson, S. Horschel, and W. Provancher. Design of a fingertipmounted tactile display with tangential skin displacement feedback. Haptics, IEEE Transactions on, 3(4):297-301, 2010.

[13] O. Goksel, S. Jeon, M. Harders, and G. Szekely. Deformable haptic model generation through manual exploration. In World Haptics Conference (WHC), 2013, pages 543-548. IEEE, 2013.

[14] G. Irving, J. Teran, and R. Fedkiw. Invertible finite elements for robust simulation of large deformation. Proc. of ACM SIGGRAPH/Eurographics Symposium on Computer Animation, pages 131-140, 2004.

[15] V. Lévesque. Measurement of Skin Deformation Using Fingerprint Feature Tracking. PhD thesis, McGill University, 2002.
[16] T. MAENO, K. KOBAYASHI, and N. YAMAZAKI. Relationship between the structure of human finger tissue and the location of tactile receptors. JSME International Journal Series C, 41(1):94-100, 1998.

[17] E. Miguel, D. Bradley, B. Thomaszewski, B. Bickel, W. Matusik, M. A. Otaduy, and S. Marschner. Data-driven estimation of cloth simulation models. Computer Graphics Forum (Proc. of Eurographics), 31(2), may 2012.

[18] K. Minamizawa, H. Kajimoto, N. Kawakami, and S. Tachi. A wearable haptic display to present the gravity sensation - preliminary observations and device design. In EuroHaptics Conference, 2007 and Symposium on Haptic Interfaces for Virtual Environment and Teleoperator Systems. World Haptics 2007. Second Joint, pages 133138, March 2007.

[19] M. Müller and M. Gross. Interactive virtual materials. Proc. of Graphics Interface, 2004.

[20] R. W. Ogden. Non-Linear Elastic Deformations. Courier Dover Publications, 1997.

[21] D. K. Pai, K. van den Doel, D. L. James, J. Lang, J. E. Lloyd, J. L. Richmond, and S. H. Yau. Scanning physical interaction behavior of 3d objects. In Proc. of ACM SIGGRAPH 2001, Computer Graphics Proc., pages 87-96, Aug. 2001.

[22] W. J. Peine and R. D. Howe. Do humans sense finger deformation or distributed pressure to detect lumps in soft tissue? Proc. of the ASME Dynamic Systems and Control Division, ASME International Mechanical Engineering Congress and Exposition, 1998.

[23] A. Perez, G. Cirio, F. Hernandez, C. Garre, and M. Otaduy. Strain limiting for soft finger contact simulation. In World Haptics Conference (WHC), 2013, pages 79-84, 2013.

[24] J. Perez, A. G. Perez, and M. A. Otaduy. Simulation of hyperelastic materials using energy constraints. In Proc. of Spanish Computer Graphics Conference, 2013.

[25] D. Prattichizzo, F. Chinello, C. Pacchierotti, and M. Malvezzi. Towards wearability in fingertip haptics: a 3-dof wearable device for cutaneous force feedback. IEEE Transactions on Haptics, 6(4):506-516, 2013.

[26] X. Provot. Deformation constraints in a mass-spring model to describe rigid cloth behavior. Proc. of Graphics Interface, 1995.

[27] J. L. Schoner, J. Lang, and H.-P. Seidel. Measurement-based interactive simulation of viscoelastic solids. Computer Graphics Forum (Proc. Eurographics), 23(3):547-556, 2004.

[28] E. R. Serina, C. D. Mote, and D. Rempel. Force response of the fingertip pulp to repeated compression: Effects of loading rate, loading angle and anthropometry. Journal of Biomechanics, 30(10), 1997.

[29] A. Serio, M. Bianchi, and A. Biechi. A device for mimicking the contact force/contact area relationship of different materials with applications to softness rendering. In Intelligent Robots and Systems (IROS), 2013 IEEE/RSJ International Conference on, pages 44844490, 2013.

[30] S. SHIMAWAKI and N. SAKAI. Quasi-static deformation analysis of a human finger using a three-dimensional finite element model constructed from ct images. Journal of Environment and Engineering, 2(1):56-63, 2007.

[31] M. Solazzi, W. Provancher, A. Frisoli, and M. Bergamasco. Design of a sma actuated 2-dof tactile device for displaying tangential skin displacement. In World Haptics Conference (WHC), 2011 IEEE, pages 31-36, 2011.

[32] W. F. SOUTHWOOD. The thickness of the skin. Plast Reconstr Surg, 15(5):423-429, May 1955.

[33] B. Thomaszewski, S. Pabst, and W. Strasser. Continuum-based strain limiting. Computer Graphics Forum, 28(2):569-576, 2009.

[34] P. Volino and N. Magnenat-Thalmann. Stop-and-go cloth draping. Vis. Comput., 23(9):669-677, 2007.

[35] H. Wang, J. O'Brien, and R. Ramamoorthi. Data-driven elastic models for cloth: Modeling and measurement. ACM Transactions on Graphics, 2011.

[36] J. Z. Wu, R. G. Dong, S. Rakheja, A. W. Schopper, and W. P. Smutz. A structural fingertip model for simulating of the biomechanics of tactile sensation. Med Eng Phys, 26(2):165-175, Mar 2004. 